\title{
A Non-PSLQ Route to BBP-type Formulas
}

\author{
Kunle Adegoke \\ Department of Physics, Obafemi Awolowo University \\ Ile-Ife, Nigeria \\ E-mail: adegoke@daad-alumni.de
}

\begin{abstract}
Starting with two simple generators, we derive a wide range of general BBP-Type formulas in arbitrary bases. Many known BBP-Type formulas are seen to be particular cases of the formulas presented.

Keywords: BBP-type formula, PSLQ algorithm

\section{Introduction}

The study of BBP-Type (Bailey, Borwein \& Plouffe, 1997) formulas has continued to attract attention, mainly because they facilitate digit extraction through a simple algorithm not requiring multiple-precision arithmetic (Bailey, 2009). Experimentally, these formulas are usually discovered by using Bailey and Ferguson's PSLQ (Partial Sum of Least Squares) algorithm (Ferguson et. al., 1999) or its variations. A drawback is that PSLQ and other integer relation finding schemes typically do not suggest proofs (Bailey, 2006). Formal proofs must be developed after the formulas have been discovered. There have been attempts in the past to give general formulas which include the proofs, as can be found, for example, in the following references: (Bellard, 1997), (Broadhurst, 1998) and (Adamchik and Wagon, 1996). In this paper we give two identities which generate a wide range of BBP-Type formulas in arbitrary bases. Many BBP-Type formulas that are known in the literature are seen to be mere particular instances of the more general formulas presented here.
\end{abstract}

\section{Generators of BBP-Type formulas}

Consider the simple Taylor formula

$$
-\ln (1-z)=\sum_{k=1}^{\infty} \frac{z^{k}}{k}
$$

valid for $|z|<1$. Choosing $z=p \cos x+\mathrm{i} p \sin x$, for real $p$ and $x$, allows one to write the left side of Eq. (1) as

$$
\begin{aligned}
-\ln (1-z) & \equiv-\ln \left[\sqrt{\left(1-2 p \cos x+p^{2}\right)} \exp \left(i \arctan \left(\frac{-p \sin x}{1-p \cos x}\right)\right)\right] \\
& =-\frac{1}{2} \ln \left(1-2 p \cos x+p^{2}\right)+i \arctan \left(\frac{p \sin x}{1-p \cos x}\right)
\end{aligned}
$$

and the right side of Eq. (1) as

$$
\sum_{k=1}^{\infty} \frac{z^{k}}{k} \equiv \sum_{k=1}^{\infty} \frac{(p \cos x+\mathrm{i} p \sin x)^{k}}{k}=\sum_{k=1}^{\infty} \frac{p^{k} \cos (k x)}{k}+\mathrm{i} \sum_{k=1}^{\infty} \frac{p^{k} \sin (k x)}{k}
$$

In Eq. (3), we used De Moiver theorem.

Equating real and imaginary parts of Eq. (2) and Eq. (3), leads to the following two identities:

$$
\arctan \left(\frac{p \sin x}{1-p \cos x}\right)=\sum_{k=1}^{\infty} \frac{p^{k} \sin (k x)}{k}
$$

and

$$
-\frac{1}{2} \ln \left(1-2 p \cos x+p^{2}\right)=\sum_{k=1}^{\infty} \frac{p^{k} \cos (k x)}{k}
$$

for $|p|<1$.

We demonstrate in the following sections that careful choices of $p$ and $x$ in Eq. (4) and Eq. (5) lead to interesting BBPType series. 


\section{Arctangent formulas}

3.1 BBP-Type formulas generated by $x=\pi / 2$ in Eq. (4)

The choice $x=\pi / 2$ in Eq. (4) gives the formula

$$
\arctan p=\sum_{k=1}^{\infty} \frac{p^{k} \sin (k \pi / 2)}{k}
$$

Since

$$
\sin \left(\frac{k \pi}{2}\right)= \begin{cases}1 & \text { if } k=1,5,9,13,17, \ldots \\ 0 & \text { if } k=2,4,6,8,10, \ldots \\ -1 & \text { if } k=3,7,11,15,19, \ldots\end{cases}
$$

Eq. (6) can be written as

$$
\arctan p=\sum_{k=0}^{\infty} p^{4 k}\left[\frac{p}{4 k+1}-\frac{p^{3}}{4 k+3}\right] .
$$

Note that Eq. (7) is convergent for $|p| \leq 1$. When $p= \pm 1$, we merely recover a rewritten version of the well-known Gregory formula for $\pi / 4$. Setting $p=1 / u$, Eq. (7) can be written

$$
u^{3} \arctan \frac{1}{u}=\sum_{k=0}^{\infty} \frac{1}{u^{4 k}}\left[\frac{u^{2}}{4 k+1}-\frac{1}{4 k+3}\right] .
$$

If $u^{2}$ is a positive integer, then Eq. (8) is a BBP-Type formula. Thus, with $u^{2}=n$, we have:

$$
\sqrt{n} \arctan \left(\frac{1}{\sqrt{n}}\right)=\frac{1}{n} \sum_{k=0}^{\infty} \frac{1}{\left(n^{2}\right)^{k}}\left[\frac{n}{4 k+1}-\frac{1}{4 k+3}\right], \quad n \in \mathbb{Z}^{+} .
$$

In the notation employed in the BBP Compendium (Bailey, 2009)

$$
\sqrt{n} \arctan \left(\frac{1}{\sqrt{n}}\right)=\frac{1}{n} P\left(1, n^{2}, 4,(n, 0,-1,0)\right) .
$$

Note that the particular case $n=2$ is a base- 4 version of formula 18 of the Compendium. To see this we write the base $n^{2}$, length 4 formula Eq. (9) as a base $n^{4}$, length 8 formula as follows:

$$
\begin{aligned}
& \sum_{k=0}^{\infty} \frac{1}{\left(n^{2}\right)^{k}}\left[\frac{n}{4 k+1}-\frac{1}{4 k+3}\right]=\sum_{k \text { even }}()+\sum_{k \text { odd }}() \\
& =\sum_{k=0}^{\infty} \frac{1}{\left(n^{2}\right)^{2 k}}\left[\frac{n}{4(2 k)+1}-\frac{1}{4(2 k)+3}\right]+\sum_{k=0}^{\infty} \frac{1}{\left(n^{2}\right)^{2 k+1}}\left[\frac{n}{4(2 k+1)+1}-\frac{1}{4(2 k+1)+3}\right] \\
& =\frac{1}{n^{2}} \sum_{k=0}^{\infty} \frac{1}{\left(n^{4}\right)^{k}}\left[\frac{n^{3}}{8 k+1}-\frac{n^{2}}{8 k+3}+\frac{n}{8 k+5}-\frac{1}{8 k+7}\right] .
\end{aligned}
$$

Thus in base $n^{4}$, length 8 we have

$$
\begin{aligned}
\sqrt{n} \arctan \left(\frac{1}{\sqrt{n}}\right) & =\frac{1}{n^{3}} \sum_{k=0}^{\infty} \frac{1}{\left(n^{4}\right)^{k}}\left[\frac{n^{3}}{8 k+1}-\frac{n^{2}}{8 k+3}+\frac{n}{8 k+5}-\frac{1}{8 k+7}\right] \\
& =\frac{1}{n^{3}} P\left(1, n^{4}, 8,\left(n^{3}, 0,-n^{2}, 0, n,-1\right)\right) .
\end{aligned}
$$

The particular case $n=2$ in Eq. (10) recovers formula 18 of the Compendium.

Similarly, the particular case $n=3$ in Eq. (9) is a base-9 length 4 version of formula 41 of the Compendium.

In general a formula with base $b$ and length $l$ can be rewritten as a formula with base $b^{r}$ and length $r l$ (Bailey, 2009).

Observe that Eq. (6) can also be written

$$
\arctan p=p \sum_{k=0}^{\infty} \frac{\left(-p^{2}\right)^{k}}{2 k+1}
$$


which gives the alternating base $n$ version of Eq. (9) as

$$
\begin{aligned}
\sqrt{n} \arctan \left(\frac{1}{\sqrt{n}}\right) & =\sum_{k=0}^{\infty} \frac{1}{(-n)^{k}}\left[\frac{1}{2 k+1}\right], \quad n \in \mathbb{Z}^{+} \\
& =P(1,-n, 2,(1,0)) .
\end{aligned}
$$

3.2 BBP-Type formulas generated by $x=\pi / 3$ in Eq. (4)

Putting $x=\pi / 3$ in Eq. (4) gives

$$
\arctan \left(\frac{p \sqrt{3}}{2-p}\right)=\sum_{k=1}^{\infty} \frac{p^{k} \sin (k \pi / 3)}{k}
$$

Noting that

$$
\sin \left(\frac{k \pi}{3}\right)=\frac{\sqrt{3}}{2} \begin{cases}1 & \mathrm{k}=1,2,7,8,13,14, \ldots \\ 0 & \mathrm{k}=0,3,6,9,12,15, \ldots \\ -1 & \mathrm{k}=4,5,10,11,16,17, \ldots\end{cases}
$$

we have the following formula:

$$
\arctan \left(\frac{p \sqrt{3}}{2-p}\right)=\frac{\sqrt{3}}{2} \sum_{k=0}^{\infty}(-1)^{k} p^{3 k}\left[\frac{p}{3 k+1}+\frac{p^{2}}{3 k+2}\right] .
$$

Eq. (11) is a BBP-formula if $p=1 / \pm n, n$ a positive integer. The choice $p=1 / n$ leads to

$$
n^{2} \arctan \left(\frac{\sqrt{3}}{2 n-1}\right)=\frac{\sqrt{3}}{2} \sum_{k=0}^{\infty} \frac{1}{\left(-n^{3}\right)^{k}}\left[\frac{n}{3 k+1}+\frac{1}{3 k+2}\right] n \in \mathbb{Z}^{+},
$$

while the choice $p=-1 / n$ gives

$$
n^{2} \arctan \left(\frac{\sqrt{3}}{2 n+1}\right)=\frac{\sqrt{3}}{2} \sum_{k=0}^{\infty} \frac{1}{\left(n^{3}\right)^{k}}\left[\frac{n}{3 k+1}-\frac{1}{3 k+2}\right] \quad n \in \mathbb{Z}^{+} .
$$

That is

$$
n^{2} \arctan \left(\frac{\sqrt{3}}{2 n-1}\right)=\frac{\sqrt{3}}{2} P\left(1,-n^{3}, 3,(n, 1,0)\right)
$$

and

$$
n^{2} \arctan \left(\frac{\sqrt{3}}{2 n+1}\right)=\frac{\sqrt{3}}{2} P\left(1, n^{3}, 3,(n,-1,0)\right) .
$$

A particular case of Eq. (13) is formula (40) in the BBP Compendium, corresponding to $n=3$ here. $n=2$ in Eq. (12) also gives a formula that is equivalent to formula (16) in the Compendium.

3.3 BBP-Type formulas generated by $x=\pi / 4$ in Eq. (4)

$x=\pi / 4$ in Eq. (4) gives

$$
\arctan \left(\frac{p}{\sqrt{2}-p}\right)=\sum_{k=1}^{\infty} \frac{p^{k} \sin (k \pi / 4)}{k}
$$

Observing that

we obtain

$$
\sin \left(\frac{k \pi}{4}\right)= \begin{cases}1 & \mathrm{k}=2,10,18,26,34, \ldots \\ 1 / \sqrt{2} & \mathrm{k}=1,3,9,11,17,19, \ldots \\ 0 & \mathrm{k}=0,4,8,12,16,20, \ldots \\ -1 / \sqrt{2} & \mathrm{k}=5,7,13,15,21,23, \ldots \\ -1 & \mathrm{k}=6,14,22,30,38,46, \ldots\end{cases}
$$

$$
\begin{array}{r}
\arctan \left(\frac{p}{\sqrt{2}-p}\right)=\sum_{k=0}^{\infty} p^{8 k}\left[\frac{p}{\sqrt{2}} \frac{1}{8 k+1}+\frac{p^{2}}{8 k+2}+\frac{p^{3}}{\sqrt{2}} \frac{1}{8 k+3}\right. \\
\left.-\frac{p^{5}}{\sqrt{2}} \frac{1}{8 k+5}-\frac{p^{6}}{8 k+6}-\frac{p^{7}}{\sqrt{2}} \frac{1}{8 k+7}\right]
\end{array}
$$


On setting $p=\sqrt{2} / u$, Eq. (14) can be written

$$
\begin{array}{r}
u^{7} \arctan \left(\frac{1}{u-1}\right)=\sum_{k=0}^{\infty} \frac{1}{(u / \sqrt{2})^{8 k}}\left[\frac{u^{6}}{8 k+1}+\frac{2 u^{5}}{8 k+2}+\frac{2 u^{4}}{8 k+3}\right. \\
\left.-\frac{4 u^{2}}{8 k+5}-\frac{8 u}{8 k+6}-\frac{8}{8 k+7}\right]
\end{array}
$$

Similarly setting $p=-\sqrt{2} / u$, Eq. (14) can be written

$$
\begin{array}{r}
u^{7} \arctan \left(\frac{1}{u+1}\right)=\sum_{k=0}^{\infty} \frac{1}{(u / \sqrt{2})^{8 k}}\left[\frac{u^{6}}{8 k+1}-\frac{2 u^{5}}{8 k+2}+\frac{2 u^{4}}{8 k+3}\right. \\
\left.-\frac{4 u^{2}}{8 k+5}+\frac{8 u}{8 k+6}-\frac{8}{8 k+7}\right]
\end{array}
$$

Although Eqs. (15) and (16) are valid for any $u \geq \sqrt{2}$, they are BBP-Type series only if $u$ is an even integer. Thus, setting $u=2 n$ in Eq. (15) and Eq. (16) respectively, we obtain the following BBP-Type formulas:

$$
\begin{aligned}
n^{7} \arctan \left(\frac{1}{2 n-1}\right)=\frac{1}{16} & \sum_{k=0}^{\infty} \frac{1}{\left(16 n^{8}\right)^{k}}\left[\frac{8 n^{6}}{8 k+1}+\frac{8 n^{5}}{8 k+2}+\frac{4 n^{4}}{8 k+3}\right. \\
& \left.-\frac{2 n^{2}}{8 k+5}-\frac{2 n}{8 k+6}-\frac{1}{8 k+7}\right], \quad n \in \mathbb{Z}^{+}
\end{aligned}
$$

and

$$
\begin{aligned}
n^{7} \arctan \left(\frac{1}{2 n+1}\right)=\frac{1}{16} & \sum_{k=0}^{\infty} \frac{1}{\left(16 n^{8}\right)^{k}}\left[\frac{8 n^{6}}{8 k+1}-\frac{8 n^{5}}{8 k+2}+\frac{4 n^{4}}{8 k+3}\right. \\
& \left.-\frac{2 n^{2}}{8 k+5}+\frac{2 n}{8 k+6}-\frac{1}{8 k+7}\right], \quad n \in \mathbb{Z}^{+}
\end{aligned}
$$

In the P-notation then,

$$
n^{7} \arctan \left(\frac{1}{2 n-1}\right)=\frac{1}{16} P\left(1,16 n^{8}, 8,\left(8 n^{6}, 8 n^{5}, 4 n^{4}, 0,-2 n^{2},-2 n,-1,0\right)\right)
$$

and

$$
n^{7} \arctan \left(\frac{1}{2 n+1}\right)=\frac{1}{16} P\left(1,16 n^{8}, 8,\left(8 n^{6},-8 n^{5}, 4 n^{4}, 0,-2 n^{2}, 2 n,-1,0\right)\right)
$$

Formula (13) of the Compendium is a particular case of Eq. (17), with $n=1$.

Adding Eq. (15) and Eq. (16), we obtain

$$
u^{7} \arctan \left(\frac{2 u}{u^{2}-2}\right)=2 \sum_{k=0}^{\infty} \frac{1}{(u / \sqrt{2})^{8 k}}\left[\frac{u^{6}}{8 k+1}+\frac{2 u^{4}}{8 k+3}-\frac{4 u^{2}}{8 k+5}-\frac{8}{8 k+7}\right]
$$

Although Eq. (19) is true for any $u \geq \sqrt{2}$, it is a BBP-series only if $u / \sqrt{2}$ is a positive integer and $u^{2}$ is an integer, that is only if $u$ is a positive even integer. Thus, setting $u^{2}=2 n$ in Eq. (19), we obtain the BBP-Type formula

$$
n^{3} \sqrt{n} \arctan \left(\frac{\sqrt{n}}{n-1} \sqrt{2}\right)=\sqrt{2} \sum_{k=0}^{\infty} \frac{1}{n^{4 k}}\left[\frac{n^{3}}{8 k+1}+\frac{n^{2}}{8 k+3}-\frac{n}{8 k+5}-\frac{1}{8 k+7}\right]
$$

That is

$$
n^{3} \sqrt{n} \arctan \left(\frac{\sqrt{n}}{n-1} \sqrt{2}\right)=\sqrt{2} P\left(1, n^{4}, 8,\left(n^{3}, 0, n^{2}, 0,-n, 0,-1,0\right)\right) .
$$

The particular case $n=2$ corresponds to formula (8) in the Compendium. Subtracting Eq. (16) from Eq. (15) gives a formula which is equivalent to Eq. (9) and therefore contains no new information. 
3.4 BBP-Type formulas generated by $x=\pi / 6$ in Eq. (4)

With $x=\pi / 6$ in Eq. (4), we have

$$
\arctan \left(\frac{2 p+p^{2} \sqrt{3}}{4-3 p^{2}}\right)=\sum_{k=1}^{\infty} \frac{p^{k} \sin (k \pi / 6)}{k}
$$

Noting that

$$
\sin \left(\frac{k \pi}{6}\right)= \begin{cases}1 & \mathrm{k}=3,15,27,39,51, \ldots \\ \sqrt{3} / 2 & \mathrm{k}=2,4,14,16,26,28, \ldots \\ 1 / 2 & \mathrm{k}=1,5,13,17,25,29,37,41 \ldots \\ 0 & \mathrm{k}=6,12,18,24,30,36 \ldots \\ -1 / 2 & \mathrm{k}=7,11,19,23,31,35, \ldots \\ -\sqrt{3} / 2 & \mathrm{k}=8,10,20,22,32,34, \ldots \\ -1 & \mathrm{k}=9,21,33,45,57, \ldots\end{cases}
$$

we obtain

$$
\arctan \left(\frac{2 p+p^{2} \sqrt{3}}{4-3 p^{2}}\right)=\sum_{k=0}^{\infty}\left(-p^{6}\right)^{k}\left[\frac{1}{2} \frac{p}{6 k+1}+\frac{\sqrt{3}}{2} \frac{p^{2}}{6 k+2}+\frac{p^{3}}{6 k+3}+\frac{\sqrt{3}}{2} \frac{p^{4}}{6 k+4}+\frac{1}{2} \frac{p^{5}}{6 k+5}\right]
$$

Setting $p=\sqrt{3} / u$ and $p=-\sqrt{3} / u$ respectively in Eq. (22) yield the following series

$$
u^{5} \arctan \left(\frac{\sqrt{3}}{2 u-3}\right)=\frac{\sqrt{3}}{2} \sum_{k=0}^{\infty} \frac{1}{\left(-u^{6} / 27\right)^{k}}\left[\frac{u^{4}}{6 k+1}+\frac{3 u^{3}}{6 k+2}+\frac{6 u^{2}}{6 k+3}+\frac{9 u}{6 k+4}+\frac{9}{6 k+5}\right]
$$

and

$$
u^{5} \arctan \left(\frac{\sqrt{3}}{2 u+3}\right)=\frac{\sqrt{3}}{2} \sum_{k=0}^{\infty} \frac{1}{\left(-u^{6} / 27\right)^{k}}\left[\frac{u^{4}}{6 k+1}-\frac{3 u^{3}}{6 k+2}+\frac{6 u^{2}}{6 k+3}-\frac{9 u}{6 k+4}+\frac{9}{6 k+5}\right]
$$

Eq. (23) and Eq. (24) converge for $u \geq \sqrt{3}$. They are however BBP-Type series only if $u$ is a multiple of 3. Thus, with $u=3 n$, we obtain the following BBP-Type series:

$$
\begin{aligned}
27 n^{5} \arctan \left(\frac{1}{\sqrt{3}} \frac{1}{2 n-1}\right) & =\frac{\sqrt{3}}{2} \sum_{k=0}^{\infty} \frac{1}{\left(-27 n^{6}\right)^{k}}\left[\frac{9 n^{4}}{6 k+1}+\frac{9 n^{3}}{6 k+2}+\frac{6 n^{2}}{6 k+3}+\frac{3 n}{6 k+4}+\frac{1}{6 k+5}\right] \\
& =\frac{\sqrt{3}}{2} P\left(1,-27 n^{6}, 6,\left(9 n^{4}, 9 n^{3}, 6 n^{2}, 3 n, 1,0\right)\right)
\end{aligned}
$$

and

$$
\begin{aligned}
27 n^{5} \arctan \left(\frac{1}{\sqrt{3}} \frac{1}{2 n+1}\right) & =\frac{\sqrt{3}}{2} \sum_{k=0}^{\infty} \frac{1}{\left(-27 n^{6}\right)^{k}}\left[\frac{9 n^{4}}{6 k+1}-\frac{9 n^{3}}{6 k+2}+\frac{6 n^{2}}{6 k+3}-\frac{3 n}{6 k+4}+\frac{1}{6 k+5}\right] \\
& =\frac{\sqrt{3}}{2} P\left(1,-27 n^{6}, 6,\left(9 n^{4},-9 n^{3}, 6 n^{2},-3 n, 1,0\right)\right)
\end{aligned}
$$

Formula (41) of the Compendium is a particular case of formula (25), corresponding to $n=1$.

Addition of Eq. (23) and Eq. (24), with $u=3 n$, gives the following BBP-Type series

$$
\begin{aligned}
n^{2} \sqrt{n} \arctan \left(\frac{\sqrt{n}}{n-1}\right) & =\sum_{k=0}^{\infty} \frac{1}{\left(-n^{3}\right)^{k}}\left[\frac{n^{2}}{6 k+1}+\frac{2 n}{6 k+3}+\frac{1}{6 k+5}\right] \\
& =P\left(1,-n^{3}, 6,\left(n^{2}, 0,2 n, 0,1,0\right)\right.
\end{aligned}
$$

Subtraction of Eq. (24) from Eq. (23) yields Eq. (12) and therefore does not give new information. 


\section{Logarithm formulas}

Using reasoning that is completely similar to that in the previous section, we present the following BBP-Type formulas for logarithm. In each formula, $n$ is a positive integer (except in formulas (29) to (31) where $n=1$ must be excluded).

4.1 BBP-Type formulas generated by $x=\pi / 2$ in Eq. (5)

$$
\begin{aligned}
\ln \left(\frac{n+1}{n}\right) & =\frac{1}{n^{2}} \sum_{k=0}^{\infty} \frac{1}{\left(n^{2}\right)^{k}}\left[\frac{n}{2 k+1}-\frac{1}{2 k+2}\right] \\
& =\frac{1}{n^{2}} P\left(1, n^{2}, 2,(n,-1)\right) . \\
\ln \left(\frac{n-1}{n}\right) & =-\frac{1}{n^{2}} \sum_{k=0}^{\infty} \frac{1}{\left(n^{2}\right)^{k}}\left[\frac{n}{2 k+1}+\frac{1}{2 k+2}\right] \\
& =-\frac{1}{n^{2}} P\left(1, n^{2}, 2,(n, 1)\right) .
\end{aligned}
$$

Addition of Eq. (28) and Eq. (29) gives

$$
\begin{aligned}
\ln \left(\frac{n-1}{n}\right) & =-\frac{1}{n} \sum_{k=0}^{\infty} \frac{1}{n^{k}}\left[\frac{1}{k+1}\right] \\
& =-\frac{1}{n} P(1, n, 1,(1)) .
\end{aligned}
$$

Fomula (47) of the BBP Compendium is a particular case of Eq. (30), with $n=10$.

Subtraction of Eq. (29) from Eq. (28) gives

$$
\begin{aligned}
\ln \left(\frac{\sqrt{n}+1}{\sqrt{n}-1}\right) & =\frac{2}{\sqrt{n}} \sum_{k=0}^{\infty} \frac{1}{n^{k}}\left[\frac{1}{2 k+1}\right] \\
& =\frac{2}{\sqrt{n}} P(1, n, 2,(1,0)) .
\end{aligned}
$$

Formula (6) of the Compendium is a particular case of Eq. (31), with $n=4$, while Formula (39) of the Compendium is another particular case, with $n=9 . n=2$ in Eq. (31) gives a length 2 version of formula (17) in the Compendium.

4.2 BBP-Type formulas generated by $x=\pi / 3$ in Eq. (5)

$$
\begin{aligned}
\ln \left(\frac{n^{2}-n+1}{n^{2}}\right) & =-\frac{1}{n^{3}} \sum_{k=0}^{\infty} \frac{1}{\left(-n^{3}\right)^{k}}\left[\frac{n^{2}}{3 k+1}-\frac{n}{3 k+2}-\frac{2}{3 k+3}\right] \\
& =-\frac{1}{n^{3}} P\left(1,-n^{3}, 3,\left(n^{2},-n,-2\right)\right) . \\
\ln \left(\frac{n^{2}+n+1}{n^{2}}\right) & =\frac{1}{n^{3}} \sum_{k=0}^{\infty} \frac{1}{\left(n^{3}\right)^{k}}\left[\frac{n^{2}}{3 k+1}+\frac{n}{3 k+2}-\frac{2}{3 k+3}\right] \\
& =\frac{1}{n^{3}} P\left(1, n^{3}, 3,\left(n^{2}, n,-2\right)\right) .
\end{aligned}
$$


4.3 BBP-Type formulas generated by $x=\pi / 4$ in Eq. (5)

$$
\begin{aligned}
\ln \left(\frac{2 n^{2}-2 n+1}{2 n^{2}}\right) & =-\frac{1}{2 n^{4}} \sum_{k=0}^{\infty} \frac{1}{\left(-4 n^{4}\right)^{k}}\left[\frac{2 n^{3}}{4 k+1}-\frac{n}{4 k+3}-\frac{1}{4 k+4}\right] \\
& =-\frac{1}{2 n^{4}} P\left(1,-4 n^{4}, 4,\left(2 n^{3}, 0,-n,-1\right)\right) . \\
\ln \left(\frac{2 n^{2}+2 n+1}{2 n^{2}}\right) & =\frac{1}{2 n^{4}} \sum_{k=0}^{\infty} \frac{1}{\left(-4 n^{4}\right)^{k}}\left[\frac{2 n^{3}}{4 k+1}-\frac{n}{4 k+3}+\frac{1}{4 k+4}\right] \\
& =\frac{1}{2 n^{4}} P\left(1,-4 n^{4}, 4,\left(2 n^{3}, 0,-n, 1\right)\right) . \\
n \frac{\sqrt{n}}{\sqrt{2}} \ln \left(\frac{n+\sqrt{2} \sqrt{n}+1}{n-\sqrt{2} \sqrt{n}+1}\right)=2 \sum_{k=0}^{\infty} \frac{1}{\left(-n^{2}\right)^{k}}\left[\frac{n}{4 k+1}-\frac{1}{4 k+3}\right] & =2 P\left(1,-n^{2}, 4,(n, 0,-1,0)\right) .
\end{aligned}
$$

Note that $n=2$ in Eq. (36) gives a binary BBP-Type formula for $\log 5$.

4.4 BBP-Type formulas generated by $x=\pi / 6$ in Eq. (5)

$$
\begin{aligned}
\ln \left(\frac{3 n^{2}-3 n+1}{3 n^{2}}\right) & =-\frac{1}{27 n^{6}} \sum_{k=0}^{\infty} \frac{1}{\left(-27 n^{6}\right)^{k}}\left[\frac{27 n^{5}}{6 k+1}+\frac{9 n^{4}}{6 k+2}-\frac{3 n^{2}}{6 k+4}-\frac{3 n}{6 k+5}-\frac{2}{6 k+6}\right] \\
& =-\frac{1}{27 n^{6}} P\left(1,-27 n^{6}, 6,\left(27 n^{5}, 9 n^{4}, 0,-3 n^{2},-3 n,-2\right)\right) . \\
\ln \left(\frac{3 n^{2}+3 n+1}{3 n^{2}}\right) & =\frac{1}{27 n^{6}} \sum_{k=0}^{\infty} \frac{1}{\left(-27 n^{6}\right)^{k}}\left[\frac{27 n^{5}}{6 k+1}-\frac{9 n^{4}}{6 k+2}+\frac{3 n^{2}}{6 k+4}-\frac{3 n}{6 k+5}+\frac{2}{6 k+6}\right] \\
& =\frac{1}{27 n^{6}} P\left(1,-27 n^{6}, 6,\left(27 n^{5},-9 n^{4}, 0,3 n^{2},-3 n, 2\right)\right) .
\end{aligned}
$$

The particular case $n=3$ of Eq. (39) is equivalent to the series obtained for $\log 7$ in reference (Adamchik and Wagon, 1996). 


\section{Conclusion}

Using elementary methods, we have derived the following BBP-type formulas in arbitrary bases.

$$
\begin{aligned}
& \sqrt{n} \arctan \left(\frac{1}{\sqrt{n}}\right)=\frac{1}{n} P\left(1, n^{2}, 4,(n, 0,-1,0)\right) \\
& n^{2} \arctan \left(\frac{\sqrt{3}}{2 n-1}\right)=\frac{\sqrt{3}}{2} P\left(1,-n^{3}, 3,(n, 1,0)\right) \\
& n^{2} \arctan \left(\frac{\sqrt{3}}{2 n+1}\right)=\frac{\sqrt{3}}{2} P\left(1, n^{3}, 3,(n,-1,0)\right) \\
& n^{7} \arctan \left(\frac{1}{2 n-1}\right)=\frac{1}{16} P\left(1,16 n^{8}, 8,\left(8 n^{6}, 8 n^{5}, 4 n^{4}, 0,-2 n^{2},-2 n,-1,0\right)\right) \\
& n^{7} \arctan \left(\frac{1}{2 n+1}\right)=\frac{1}{16} P\left(1,16 n^{8}, 8,\left(8 n^{6},-8 n^{5}, 4 n^{4}, 0,-2 n^{2}, 2 n,-1,0\right)\right) \\
& n^{3} \sqrt{n} \arctan \left(\frac{\sqrt{n}}{n-1} \sqrt{2}\right)=\sqrt{2} P\left(1, n^{4}, 8,\left(n^{3}, 0, n^{2}, 0,-n, 0,-1,0\right)\right) \\
& 27 n^{5} \arctan \left(\frac{1}{\sqrt{3}} \frac{1}{2 n-1}\right)=\frac{\sqrt{3}}{2} P\left(1,-27 n^{6}, 6,\left(9 n^{4}, 9 n^{3}, 6 n^{2}, 3 n, 1,0\right)\right) \\
& 27 n^{5} \arctan \left(\frac{1}{\sqrt{3}} \frac{1}{2 n+1}\right)=\frac{\sqrt{3}}{2} P\left(1,-27 n^{6}, 6,\left(9 n^{4},-9 n^{3}, 6 n^{2},-3 n, 1,0\right)\right) \\
& n^{2} \sqrt{n} \arctan \left(\frac{\sqrt{n}}{n-1}\right)=P\left(1,-n^{3}, 6,\left(n^{2}, 0,2 n, 0,1,0\right)\right. \\
& \ln \left(\frac{n+1}{n}\right)=\frac{1}{n^{2}} P\left(1, n^{2}, 2,(n,-1)\right) \\
& \ln \left(\frac{n-1}{n}\right)=-\frac{1}{n^{2}} P\left(1, n^{2}, 2,(n, 1)\right) \\
& \ln \left(\frac{\sqrt{n}+1}{\sqrt{n}-1}\right)=\frac{2}{\sqrt{n}} P(1, n, 2,(1,0)) \\
& \ln \left(\frac{n^{2}+n+1}{n^{2}}\right)=\frac{1}{n^{3}} P\left(1, n^{3}, 3,\left(n^{2}, n,-2\right)\right) \\
& \ln \left(\frac{n^{2}-n+1}{n^{2}}\right)=-\frac{1}{n^{3}} P\left(1,-n^{3}, 3,\left(n^{2},-n,-2\right)\right) \\
& \ln \left(\frac{2 n^{2}-2 n+1}{2 n^{2}}\right)=-\frac{1}{2 n^{4}} P\left(1,-4 n^{4}, 4,\left(2 n^{3}, 0,-n,-1\right)\right) \\
& \ln \left(\frac{2 n^{2}+2 n+1}{2 n^{2}}\right)=\frac{1}{2 n^{4}} P\left(1,-4 n^{4}, 4,\left(2 n^{3}, 0,-n, 1\right)\right) \\
& n \frac{\sqrt{n}}{\sqrt{2}} \ln \left(\frac{n+\sqrt{2} \sqrt{n}+1}{n-\sqrt{2} \sqrt{n}+1}\right)=2 P\left(1,-n^{2}, 4,(n, 0,-1,0)\right) \\
& \ln \left(\frac{3 n^{2} \pm 3 n+1}{3 n^{2}}\right)= \pm \frac{1}{27 n^{6}} P\left(1,-27 n^{6}, 6,\left(27 n^{5}, \mp 9 n^{4}, 0, \pm 3 n^{2},-3 n, \pm 2\right)\right) \\
& n^{2} \frac{\sqrt{n}}{\sqrt{3}} \ln \left(\frac{n+\sqrt{3} \sqrt{n}+1}{n-\sqrt{3} \sqrt{n}+1}\right)=2 P\left(1,-n^{3}, 6,\left(n^{2}, 0,0,0,-1,0\right)\right)
\end{aligned}
$$

In the above formulas $n \in \mathbb{Z}^{+}$.

\section{References}

Bailey, David H. (2006). Algorithms for Experimental Mathematics I. [Online] Available: http://crd.lbl.gov/ dhbailey/dhbpapers/

Bailey, David H. (2009). A compendium of bbp-type formulas for mathematical constants. [Online] Available: http://crd. lbl.gov/dhbailey/dhbpapers/bbp-formulas.pdf

David H. Bailey, Peter B. Borwein, \& Simon Plouffe. (1997). On the rapid computation of various polylogarithmic constants. Mathematics of Computation, 66 (218):903-913. 
D. J. Broadhurst. (1998). Polylogarithmic ladders, hypergeometric series and the ten millionth digits of $\zeta(3)$ and $\zeta(5)$. arXiv:math/9803067v1 [math.CA]

F. Bellard. (1997). A new formula to compute the nth binary digit of pi. [Online] Available: http://www-stud.enst.fr/bell ard/pi/pi_bin/pi_bin.html

H. R. P. Ferguson, D. H. Bailey, \& S. Arno. (1999). Analysis of PSLQ, an integer relation finding algorithm. Mathematics of Computation, 68:351-369.

Victor Adamchik and Stan Wagon. (1996). Pi: A 2000-year-old search changes direction. Mathematica in Science and Education, 5:11-19. 